In der Rubrik „Literatur kompakt" werden die wichtigsten Originalarbeiten aus der internationalen Fachliteratur referiert.

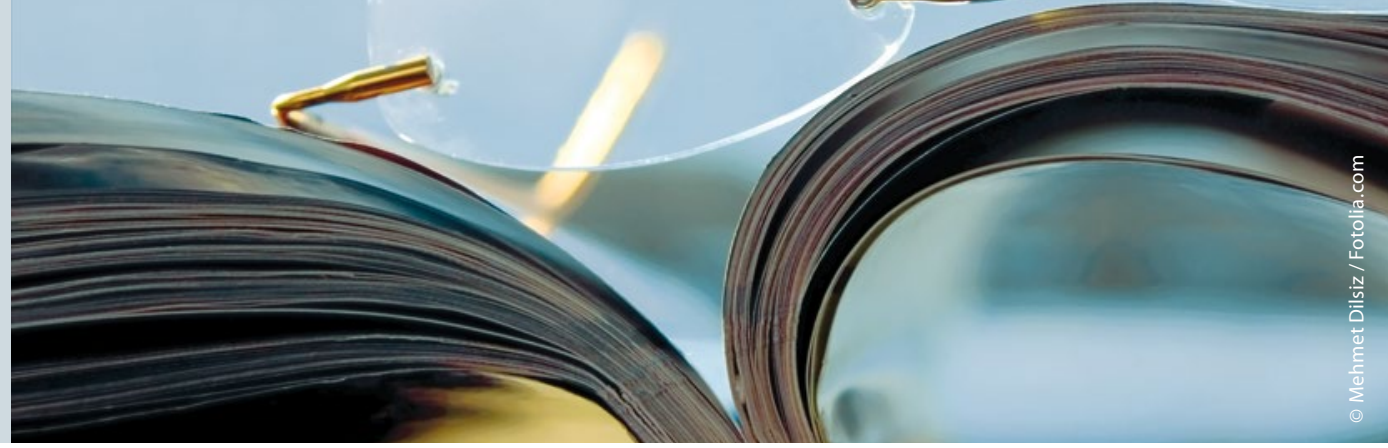

\section{Off-pump-Bypassoperation langfristig ohne Vorteile}

Die Bypassoperation am schlagenden Herzen ohne Herz-LungenMaschine (off pump) zeigt keinen Vorteil im Langzeitverlauf über fünf Jahre im Vergleich zur Operation unter Verwendung der HerzLungen-Maschine (on pump).

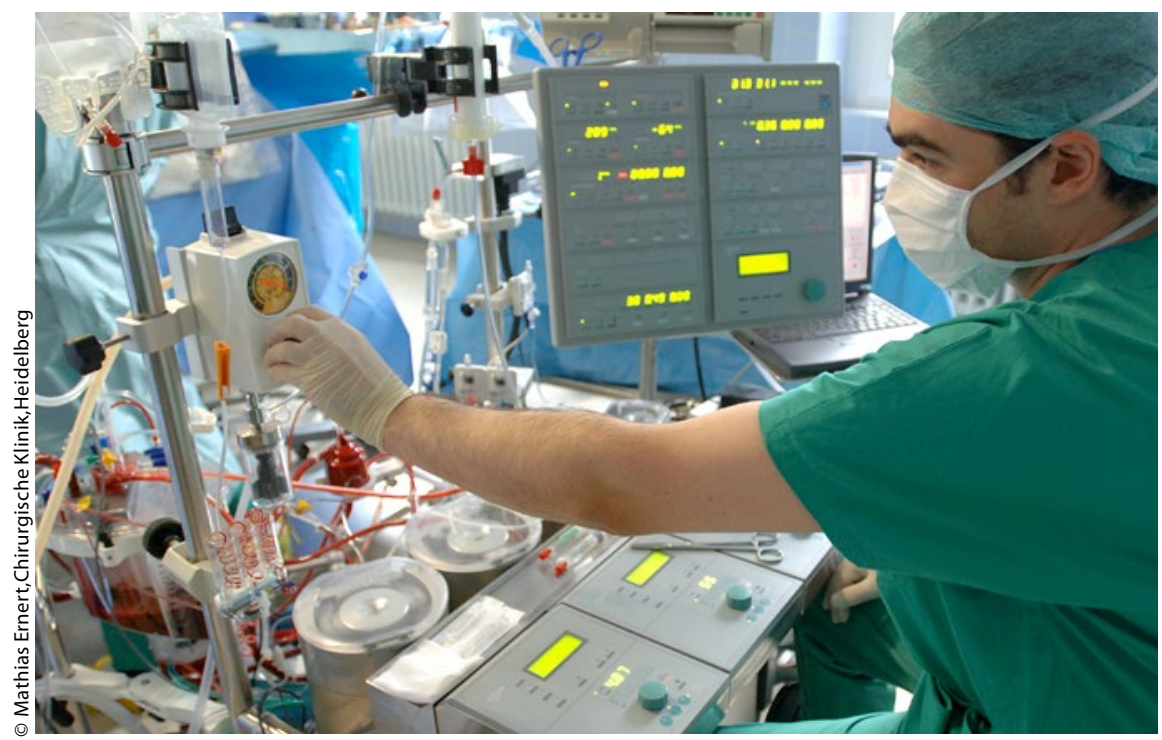

Der Einsatz einer Herz-Lungen-Maschine bei Bypassoperationen scheint im Vergleich zur Off-pump-Technik nicht nachteilig zu sein.

$B^{c}$ ei der Mehrzahl der Patienten mit koronarer Herzerkrankung wird im Rahmen einer aortokoronaren Bypassoperation eine Herz-Lungen-Maschine eingesetzt. Das perioperative Risiko zu sterben beträgt rund $2 \%$, wobei dieses bei Risikokollektiven (z. B. Patienten mit Myokardinfarkt oder schwerer Niereninsuffizienz) um das 2- bis 5-Fache erhöht sein kann. Die Entwicklung von
Techniken, Bypassoperationen auch am schlagenden Herzen und ohne Verwendung der Herz-Lungen-Maschine durchzuführen (off pump) sollte zur Verringerung periprozeduraler Komplikationen sowie Verbesserung der Langzeitergebnisse führen. Auch wenn mehrere randomisierte Studien zum Vergleich vorliegen, wurden die Langzeitergebnisse bislang noch nicht untersucht.
Nun wurden die Patienten der bislang größten Vergleichsstudie zwischen einem Off-pump- und On-pump-Vorgehen bei Bypassoperationen (CORONARY-Studie, initial 4.752 Patienten randomisiert) über fünf Jahre lang nachbeobachtet. Komponenten des primären Endpunkts waren das Auftreten von Tod, Schlaganfall, Myokardinfarkt und Nierenversagen.

Im primären Endpunkt gab es zwischen beiden Behandlungsgruppen keinen Unterschied. Auch der sekundäre Endpunkt (Kosten in US-Dollar) war in den beiden Gruppen nicht signifikant unterschiedlich. Ebenso gab es keine Unterschiede hinsichtlich mehrerer Dimensionen der Lebensqualität.

Lamy A et al. Five-Year Outcomes after OffPump or On-Pump Coronary-Artery Bypass Grafting. N Engl J Med. 2016 Oct 23; DOI: 10.1056/NEJMoa1601564

\section{Kommentar}

Die Langzeitergebnisse der CORONARYStudie zeigen, dass sich die prozeduralen Vorteile der Off-pump-Technik (Verzicht auf Herz-Lungen-Maschine und Abklemmen der Aorta) nicht in einen klinischen Vorteil übersetzen lassen. Die Auswahl des Operationsverfahrens sollte sich daher vor allem auch nach der Erfahrung des Operateurs und/oder des Zentrums richten.

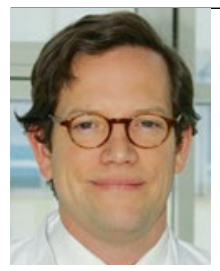

Prof. Dr. Peter W. Radke Klinik für Innere Medizin Kardiologie, Schön Klinik Neustadt 